\title{
ReaR

\section{Manejo de la vía aérea en el paciente con traumatismos sobre la vía aérea}

Artículo original: Mercer SJ, Jones CP, Bridge M, Clitheroe E, Morton B, Groom P. “Systematic Review of the anaesthetic management of non-iatrogenic acute adult airway trauma".Br J Anaesth. 2016 Sep;117 Suppl 1:i49-i59. doi: 10.1093/bja/aew193. (PubMed) (

Serna Gandía $M B$.

\section{Hospital Marina Salud Denia, Alicante.}

\section{Resumen}

Un mal manejo de la vía aérea (VA) traumatizada puede resultar catastrófica, empeorando la situación del paciente si se provoca por ejemplo, una falsa vía. Esta revisión pretende informar de la mejor práctica y sugiere diferentes opciones para el manejo de estos pacientes, en función del mecanismo traumático.

\section{Introducción}

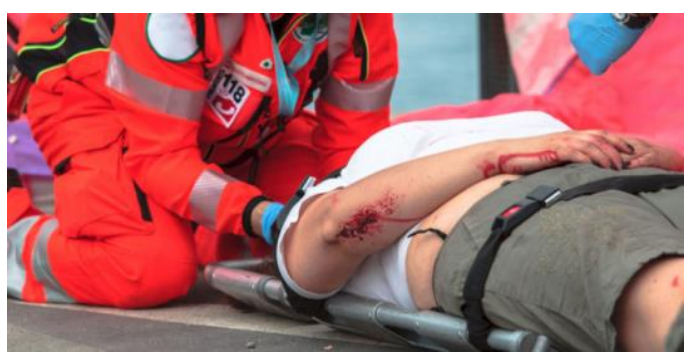

Un mal manejo de la vía aérea (VA) traumatizada puede resultar catastrófica, empeorando la situación del paciente si se provoca por ejemplo, una falsa vía. Esta revisión pretende informar de la mejor práctica y sugiere diferentes opciones para el manejo de estos pacientes, en función del mecanismo traumático.

Las lesiones provocadas por traumatismos no iatrogénicos (laceración, obstrucción, hemorragia y/o aspiración de sangre) son poco frecuentes, pudiendo constituir un reto para el anestesiólogo. A pesar de que las guías actuales (1) hablan del manejo de la vía aérea difícil (VAD) no anticipada, no abordan el manejo de la "VAD anticipada", que ocurre en estos casos. Un mal manejo de la vía aérea (VA) traumatizada puede resultar catastrófica, empeorando la situación del paciente si se provoca por ejemplo, una falsa vía. Esta revisión pretende informar de la mejor práctica y sugiere diferentes opciones para el manejo de estos pacientes, en función del mecanismo traumático.

\section{Resumen}

Los autores buscaron en Embase, Medline y Google Scholar artículos que reportaran pacientes que sufrieran traumatismos en la VA y su manejo anestésico, publicados desde el año 2000. Obtuvieron 578 resultados, cuyos abstract y títulos fueron valorados por dos revisores independientes. Tras aplicar los criterios de exclusión, se analizaron 35 publicaciones.

Los resultados fueron presentados en función del mecanismo traumático: 


\section{Traumatismo cerrado}

Los traumatismos cerrados se producen habitualmente por impactos de alta energía, como caídas desde una elevada altura, aplastamientos, colisiones de tráfico, atropellamientos, ahorcamientos o estrangulaciones.

El traumatismo maxilofacial es el mecanismo más frecuente de lesión de la VA. Puede asociar la presencia de un trismus, que normalmente se resuelve tras la inducción anestésica. En estos casos, suponen un mayor problema la presencia de hemorragia, hipoxia y el riesgo de aspiración.

Lesiones en el cartílago cricoides y en la membrana cricotiroidea ocurren en el $50 \%$ de los casos en los que hay compromiso de la VA y la separación laringotraqueal hasta en un $63 \%$. La VA puede permanecer permeable si se mantiene la ventilación espontánea debido a la contención mecánica del tejido conectivo de alrededor, aunque la situación se puede deteriorar rápidamente.

Los traumatismos a nivel de la laringe pueden presentar síntomas inespecíficos como tos, disnea, afonía, estridor, crepitantes laríngeos, hemoptisis o enfisema subcutáneo. Tanto el estridor como la hemoptipsis se han relacionado con lesiones severas.

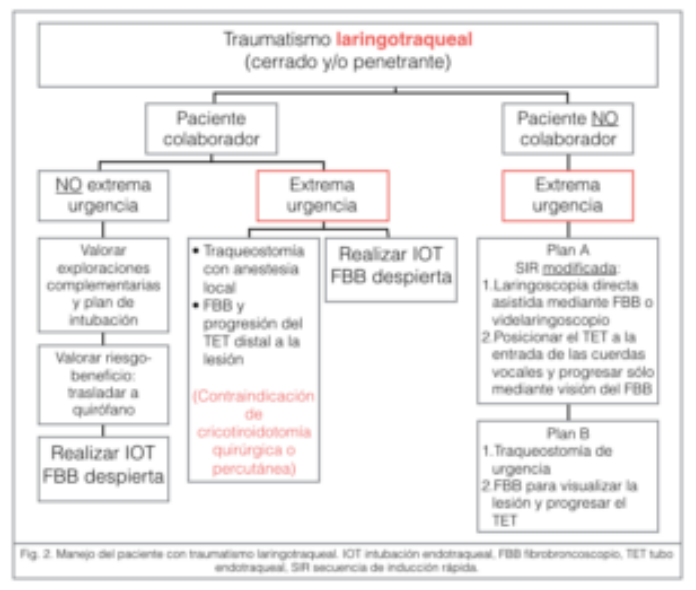

Dada la inespecificidad de los signos y síntomas, la sospecha de lesiones sobre la VA, debe llevar a la realización de exploraciones complementarias como la radiografía de tórax, en busca de un enfisema o neumotórax asociado. La endoscopia nasal permite valorar la permeabilidad de la VA superior y de las cuerdas vocales, siendo la broncoscopia necesaria para descartar lesiones por debajo de éstas. El TAC es el "gold standard" en estos casos detectando el 94\% de las lesiones.

\section{Tratamiento}

Los traumatismos cerrados menores que no requieran una actuación inmediata, deben ingresar en una unidad de cuidados intensivos para poder evaluar periódicamente la VA, durante 48 horas.

El manejo del paciente con un traumatismo grave dependerá de la cooperación de éste y de la valoración riesgo-beneficio de cada caso. La mejor opción de intubación es mediante una técnica de visualización directa y con el paciente despierto, en ventilación espontánea. Las opciones son: - Traqueostomía quirúrgica bajo anestesia local. Tanto la cricotiroidotomía quirúrgica como la percutánea están contraindicadas en estos casos.

- Intubación fibrobroncoscópica con el paciente despierto. Permite colocar el tubo endotraqueal (TET) distal a la lesión. Se debe utilizar un TET de pequeño calibre y con el bisel romo para evitar lesiones secundarias a la progresión del tubo a través del fibrobroncoscopio.

- Intubación orotraqueal convencional.

Los autores recomiendan la intubación directa mediante el uso de un fibrobroncoscopio o videolaringoscopio tras una inducción de secuencia rápida modificada (evitando el uso de presión cricoidea - maniobra de BURP - y la ventilación mediante presión positiva). 
La técnica utilizada, dependerá del grado de experiencia del anestesiólogo, del cirujano y de las condiciones del paciente.

\section{Lesiones penetrantes 0 debidas a ondas expansivas.}

Las heridas ocasionadas en la VA pueden rápidamente suponer un riesgo vital debido a la elevada densidad de estructuras vitales localizadas en el cuello. Además una lesión superficial puede esconder lesiones subyacentes vasculares, sobre la VA o el tubo digestivo. La pérdida de sangre y la obstrucción de la VA van a ser los determinantes de la severidad de la lesión en cada caso.

Las heridas causadas en la parte anterior del cuello comprimen la VA con mayor frecuencia. Si es posible, el TAC es la exploración de primera línea en pacientes con lesiones en el cuello y estables hemodinámicamente, para localizar lesiones que afecten a la VA.

En todos los casos, el manejo de estos pacientes viene determinado, al igual que en el caso anterior, por la cooperación del paciente y la valoración riesgo-beneficio. Una dificultad añadida podría suponer la presencia de hematoma o de enfisema alrededor de la VA que distorsione la anatomía y dificulte la traqueostomía. La realización de una fibrobroncoscopia puede resultar dificultosa por la presencia de sangre o detritus en la VA. A pesar de ello, en algunos casos se ha mostrado una técnica eficaz.

\section{Tratamiento}

La literatura sugiere que la opción más segura de manejo de estos pacientes es el instrumentar la tráquea bajo visión directa con el paciente despierto y en ventilación espontánea, para evitar así cualquier lesión añadida o la creación de una falsa vía. La intervención de elección es la realización de una traqueostomía quirúrgica. Tanto la cricotiroidotomía quirúrgica como la percutánea, también están contraindicadas en estos casos. La intubación fibrobroncoscópica es una alternativa, que además permite posicionar el TET en caso de que exista una lesión, distal a ésta.

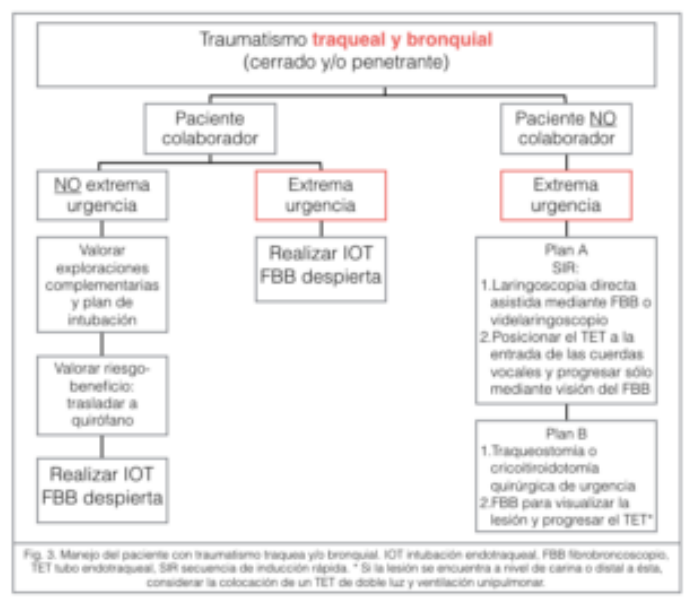

Los autores sugieren que la mejor opción es la visualización e intubación mediante el uso de fibrobroncoscopia o videolaringoscopia directa tras una inducción de secuencia rápida modificada. El uso de un AirwayScope combinado con una guía, el Airtraq o el estilete luminoso, han sido también descritos en estas situaciones.

\section{Quemaduras.}

Las quemaduras pueden ser causadas por diferentes mecanismos: vapor, calor directo, agentes corrosivos o eléctricos. Todos ellos pueden provocar una grave inflamación de la cara, lengua, epiglotis y glotis, dando lugar a una obstrucción de la VA.

Las lesiones térmicas suelen afectar a estructuras por encima de las cuerdas vocales, a no ser que se haya inhalado vapor. La inhalación de humo afecta a su vez a los pulmones, debiendo descartar en esos casos, la intoxicación por monóxido de carbono. 


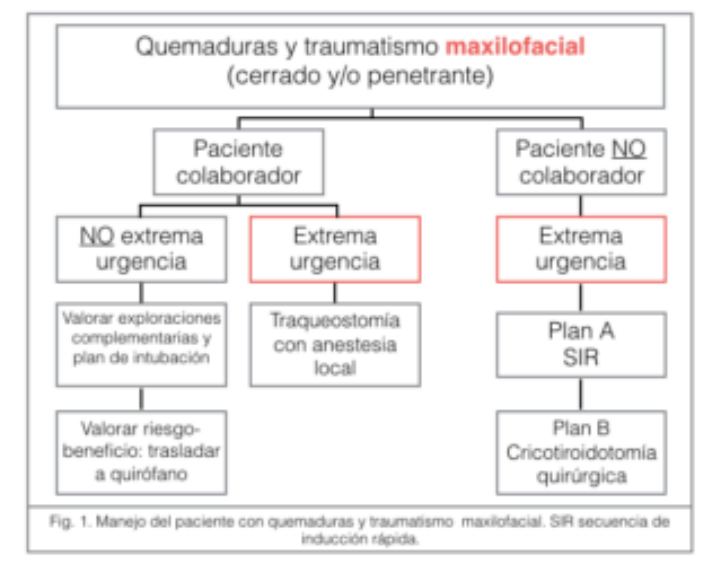

Las quemaduras sobre la cara y cuello asocian obstrucción de la VA e inhalación de humo. Debido a ello, la intubación en estos pacientes debe ser precoz, ya que la aparición de complicaciones se incrementa de un 11,2 a un $16.9 \%$ si se retrasa, debido a la aparición de edema. Se debe realizar una endoscopia nasal para valorar la severidad de las lesiones y si se sospecha inhalación de humo, una fibrobroncoscopia que pueda evidenciar la presencia de detritus, eritema o ulceraciones traqueales.

Tratamiento

La intubación está indicada en casos de lesiones por inhalación de humo combinada con quemaduras corporales extensas, de cara o cuello. Pacientes con inhalación de humo, sin presencia de quemaduras, pueden ser monitorizados mediante endoscopios nasales $\mathrm{e}$ intubarse en un segundo tiempo, en caso de ser necesario.

Además de la presencia de edema, se asocian a limitación de la apertura bucal y a un trismus, si la causa de la lesión ha sido por electrocución. La ventilación manual en estos casos también puede resultar dificultosa debido a la presencia de apósitos y exudados. En cualquier caso la administración de oxígeno se recomienda durante el manejo.

Las lesiones menores pueden tratarse de forma conservadora. En los pacientes colaboradores con quemaduras que requieran tratamiento inmediato, se debe considerar la realización de una intubación con fibrobroncoscopio despierto si la valoración previa revela la presencia de lesiones en la VA superior o dificultad de ventilación manual mediante mascarilla.

Las lesiones graves y en casos en los que el paciente no obedezca, requieren un abordaje quirúrgico de la vía aérea.

En caso de que el paciente no colabore o presente lesiones menores, la realización de una secuencia de inducción rápida seguida de intubación mediante videolaringoscopia resulta apropiada.

Tras la intubación, el TET se debe fijar cuidadosamente mediante una fijación dental o mediante sistemas de fijación por arco. Nunca se deben recortar los tubos, pues el edema facial puede provocar que éste se movilice y que el paciente requiera una reintubación.

\section{Comentario y Conclusión}

Las recomendaciones sobre el manejo de los pacientes que presentan un traumatismo de la vía aérea no iatrogénico, a diferencia de las publicadas en la última guía de la Difficult Airway Society 2015, son: - Mantenimiento de la ventilación espontánea

- Intubación mediante sistemas de visualización directa de la vía aérea para evitar la iatrogenia y la creación de una falsa vía.

- Evitar la ventilación por presión positiva y la presión cricoidea durante la secuencia de inducción rápida.

Por consiguiente, en caso de que el manejo inicial de la vía aérea fracase, únicamente se debería rescatar mediante una traqueostomía quirúrgica, para 
evitar empeorar aún más la situación del paciente.

Cuando el especialista se enfrenta a cualquier lesión traumática sobre la vía aérea, se plantean tres situaciones en función del tiempo disponible: - Sin tiempo. En este caso el anestesiólogo se pondrá en el peor de los casos y manejará la situación como tal.

-Poco tiempo.

- Suficiente tiempo como para realizar una adecuada valoración de la vía aérea.

En la mayoría de los casos la situación es intermedia, en cuyo caso es importante interactuar con el paciente y permitirle adoptar una posición "cómoda" para así "comprar" el suficiente tiempo como para hacer una adecuada valoración de la vía aérea (realizar una endoscopio nasal o un TAC), y trasladarlo a un escenario más preparado, como podría ser el quirófano.

El factor humano es fundamental en el manejo de la vía aérea difícil anticipada. El trabajo en equipo es crucial. Se tarda de 10 a 15 minutos el preparar el material para manejar una vía aérea difícil por un equipo entrenado. Durante ese tiempo el anestesiólogo debe valorar la vía aérea, planificar su manejo y considerar la necesidad de realizar exploraciones complementarias.

Por último, se debe considerar la adquisición de los conocimientos sobre el manejo de la vía aérea a partir de escenarios simulados en talleres de entrenamiento específicamente diseñados para estas situaciones.

\section{Bibliografía}

1. Hagberg CA, Gabel JC, Connis RT. Difficult Airway Society 2015 guidelines for the management of unanticipated difficult intubation in adults: not just another algorithm. Br J Anaesth. 2015 Dec;115(6):812-4. doi: 10.1093/bja/aev404. Epub 2015 Nov 10. (PubMed) ( $\underline{\text { HTML) }}$ (PDF)

Correspondencia al autor

María Beatriz Serna Gandía mariabeatriz.serna@marinasalud.es

FEA Servicio de Anestesiología y Cuidados Intensivos.

Hospital Marina Salud Denia, Alicante.

Aceptado para blog en agosto de 2017. 